\title{
Establishing research units on pediatric psychopharmacology in Brazil
}

In North America it is estimated that $10 \%$ of children and adolescents have a serious psychiatric disorder (Surgeon General Report). The term serious implies that the psychiatric disorder is a source of major impairment within the family, in the social milieu, and, very likely, in educational domain as well. Based on recent data from Brasil, there is good reason to believe that the prevalence of serious psychiatric disorders in children is similar in South America. ${ }^{1}$ Against this backdrop of documented disability associated with psychiatric disorders in childhood, there have been tremendous advances in neuroscience suggesting that at least some mental illnesses are biologically based. For example, structural MRI studies have shown smaller mean brain volumes in the dorsal lateral prefrontal region in boys with ADHD compared to controls. ${ }^{2}$ Functional MRI studies, which focus on brain function rather than structure, have shown that individuals with autism have impaired facial recognition compared to controls with similar IQ. ${ }^{3}$ To date, these advances have not been translated into treatment strategies, but we believe that in a near future neuroimaging techniques may be informative for treatment strategies.

Autism and related Pervasive Developmental Disorders (PDD) are serious psychiatric conditions of childhood onset characterized by impairments in social interaction, delayed and deviant language, and repetitive behaviors. Recent estimates from community surveys suggest that autism and PDD affect 6 children per 1,000. The most common forms of treatment for children with autism and PDD include special education, behavior therapy and medication. The aim of special education is to promote the highest degree of independence possible for each individual child. In addition to the core symptoms of repetitive behavior and impaired socialization and communication, these children are at high risk for behavioral problems including aggression, self-injury, property destruction, angry outbursts, hyperactivity and mood instability. The presence of these additional problems can interfere with other rehabilitative efforts and lead to greater long-term disability. It is often for these additional problems that medications and/or behavior therapy are given to children with
PDD. An impressive range of medications from multiple classes has been evaluated in the treatment of autism and PDD. Unfortunately, most studies were conducted in small, poorly characterized samples leaving the practicing clinician with insufficient guidance on how to match patients with a given treatment. Recognizing this lag in clinical research, the National Institute of Mental Health in the United States launched the Research Units on Pediatric Psychopharmacology (RUPP) Autism Network in 1998. The multisite network, which includes investigators from Yale University, University of California at Los Angeles, Ohio State University, Johns Hopkins University, Indiana University and the National Institute of Mental Health, has set a new standard for the design and conduct of psychopharmacology trials in children with PDD. ${ }^{4}$

Given that the goal of clinical research is to provide clear guidance to practicing clinicians on the choice of treatments, multisite trials have much to offer in pursuit of that goal. Large-scale, multisite trials are capable of providing answers to clinical questions in a timely fashion because they can enroll subjects at a faster rate that can be achieved in a single site study. This is especially important for disorders such as autism and PDD, which are serious, but not that common. For example, the RUPP Autism Network (2002) recently reported on the use of risperidone in 101 children with autism accompanied by aggression, tantrums or self-injury. This was the largest clinical trial in autism to date and the enrollment was accomplished in less than two years. The same study conducted at a single site with the same rate of recruitment would have taken 10 years. To extend this collaborative network, investigators at Yale University, Mackenzie University in São Paulo and Federal University in Bahia have taken initial steps toward establishing an international multisite network for conducting treatment studies in children and adolescents with autism and PDD.

The first step in this project will be translating valid and reliable rating instruments used in the detection and assessment of children with PDD. To improve the detection of children with developmental disorders, we have proposed a series of training programs for pediatric and family medi- 
cine practitioners. These presentations will define the various developmental disorders and provide practical screening methods that are applicable in primary care. Another series of presentations will focus on community psychiatrists, psychologists and mental health practitioners. These workshops will cover comprehensive assessment methods and evidence based treatments that are relevant for clinicians in mental health settings. In addition to the use of specific assessment instruments, these training sessions will review behavioral and psychopharmacological interventions. Finally, we will build an interdisciplinary team at each Brazilian center capable of carrying out rigorous clinical research in children with developmental disorders. This model of detection, dissemination of evidence based treatment and capacity to conduct clinical research in autism is an important first step that can set the stage for applying these efforts in other serious disorders of childhood.

Lawrence Scahill

Yale University. New Haven, CT, USA

Marcos T. Mercadante

Mackenzie Presbyterian University. São Paulo, SP, Brazil

Maria Rosario-Campos

Federal University of Bahia. Salvador, BA, Brazil

Silvia Fonseca

Hospital São Francisco. Ribeirão Preto, SP, Brazil

\section{References}

1. Fleitlich B, Goodman R. Social factors associated with child mental health problems in Brazil: cross sectional survey. BMJ 2001;323(7313):599-600.

2. Castellanos FX, Lee PP, Sharp W, Jeffries NO, Greenstein DK, Clasen LS et al. Developmental trajectories of brain volume abnormalities in children and adolescents with attention-deficit/hyperactivity disorder. JAMA 2002;288(14):1740-8.

3. Schultz RT, Gauthier I, Klin A, Fulbright RK, Anderson AW, Volkmar F et al. Abnormal ventral temporal cortical activity during face discrimination among individuals with autism and Asperger syndrome. Arch Gen Psychiatry 2000;57(4):331-40.

4. McCracken JT, McGough J, Shah B, Cronin P, Hong D, Scahill L et al. Risperidone in children with autism for serious behavioral problems. N Engl J Med 2002;347(5):314-21. 